\title{
Clinical phenotype of ASD-associated DYRK1A haploinsufficiency
}

\author{
Rachel K. Earl' ${ }^{1}$, Tychele N. Turner², Heather C. Mefford ${ }^{3}$, Caitlin M. Hudac', Jennifer Gerdts', Evan E. Eichler²,4 \\ and Raphael A. Bernier ${ }^{1,5^{*}}$
}

\begin{abstract}
Background: DYRK1A is a gene recurrently disrupted in $0.1-0.5 \%$ of the ASD population. A growing number of case reports with DYRK1A haploinsufficiency exhibit common phenotypic features including microcephaly, intellectual disability, speech delay, and facial dysmorphisms.

Methods: Phenotypic information from previously published DYRK1A cases $(n=51)$ and participants in an ongoing study at the University of Washington (UW, $n=10$ ) were compiled. Frequencies of recurrent phenotypic features in this population were compared to features observed in a large sample with idiopathic ASD from the Simons Simplex Collection ( $n=1981$ ). UW DYRK1A cases were further characterized quantitatively and compared to a randomly subsampled set of idiopathic ASD cases matched on age and gender $(n=10)$ and to cases with an ASD-associated disruptive mutation to CHD8 $(n=12)$. Contribution of familial genetic background to clinical heterogeneity was assessed by comparing head circumference, IQ, and ASD-related symptoms of UW DYRK1A cases to their unaffected parents.

Results: DYRK1A haploinsufficiency results in a common phenotypic profile including intellectual disability, speech and motor difficulties, microcephaly, feeding difficulties, and vision abnormalities. Eighty-nine percent of DYRK1A cases ascertained for ASD presented with a constellation of five or more of these symptoms. When compared quantitatively, DYRK TA cases presented with significantly lower IQ and adaptive functioning compared to idiopathic cases and significantly smaller head size compared to both idiopathic and CHD8 cases. Phenotypic variability in parental head circumference, IQ, and ASD-related symptoms corresponded to observed variability in affected child phenotype.

Conclusions: Results confirm a core clinical phenotype for DYRK1A disruptions, with a combination of features that is distinct from idiopathic ASD. Cases with DYRK1A mutations are also distinguishable from disruptive mutations to CHD 8 by head size. Measurable, quantitative characterization of DYRK1A haploinsufficiency illuminates clinical variability, which may be, in part, due to familial genetic background.
\end{abstract}

Keywords: Autism, DYRK1A, Genetic syndrome, Genetically defined subtype, Disruptive mutation, Clinical phenotype

\section{Background}

Autism spectrum disorder (ASD) is characterized by tremendous clinical variability and causal heterogeneity. Historical efforts to behaviorally subtype ASD have been largely unsuccessful due to lack of meaningful treatment implications by subtype and inadequate consensus regarding clinical phenotype $[1,2]$. Recent efforts have targeted the genetic causes of ASD to explore biologically

\footnotetext{
*Correspondence: rab2@u.washington.edu; rab2@uw.edu

'Department of Psychiatry and Behavioral Sciences, University of

Washington, CHDD Box 357920, Seattle, WA 98195, USA

${ }^{5}$ Center on Human Development and Disability, University of Washington,

Seattle, WA, USA

Full list of author information is available at the end of the article
}

defined subtypes $[3,4]$. Advances in genetic sequencing technology have improved our ability to identify diseasecausing mutations [5]. Chromosomal abnormalities, copy number variants (CNVs), and disruptive single nucleotide variants (SNVs), including nonsense, frameshift, and splice site mutations, have been associated with increased risk of ASD [6-9]. Most recently, work relating ASD risk to de novo disruptive SNVs suggests these single point mutations account for approximately $10 \%$ of ASD cases $[6,8]$. These discoveries have prompted a shift in ASD research; instead of using extensive phenotyping prior to sequencing ASD populations, researchers have begun by identifying genes of 
interest in affected individuals and then exploring phenotype in specific gene cohorts [10].

The application of this genetics-first approach to subtyping ASD has successfully identified similar medical, behavioral, and dysmorphic features shared by individuals with disruptive variants in high-confidence ASD risk genes, such as CHD8, ADNP, SCN2A, and DYRK1A (e.g., [11-13]). Dual-specificity tyrosine phosphorylationregulated kinase $1 \mathrm{~A}$, or $D Y R K 1 A$, is a highly conserved gene in the Down syndrome critical region of chromosome $21[6,14]$, and appears to play a major role in brain development, specifically neurogenesis, neural plasticity, and cellular death [15]. DYRK1A haploinsufficiency was initially identified for its role in intellectual disability, which is clinically defined as childhood onset of significant cognitive and adaptive impairment [16]. Recurrent disruptions to DYRK1A have been found in as many as $0.5 \%$ of cases with ASD [14, 17]. In Drosophila models, truncating mutations to DYRK1A (Drosophila ortholog termed the Minibrain $(M n b)$ gene) result in microcephaly, including intact but smaller brain structures [18]. Dyrk1A-null mouse models $(-/-)$ displayed growth deficiencies resulting in mid-gestational death [19]. Mice heterozygous for Dyrk1A (+/-) survived to adulthood but presented with reduced growth, developmental delays, motor and learning difficulties, and atypical behaviors, including anxiety [19, 20]. A consistent clinical phenotype appears in humans. Reported cases to date have exhibited microcephaly and intellectual disability; other features, including seizures, speech and motor delays, feeding difficulties, and distinct facial dysmorphology, have been noted as well [13, 15, 21-24].

Studies relying on medical record review have reported ASD diagnoses in up to $40 \%$ of cases with DYRK1A mutations, but many remaining cases have features consistent with ASD, such as stereotypies, reduced eye contact, and social anxiety $[15,21]$. Few studies have conducted diagnostic evaluations of ASD and ASD-related symptoms as part of a clinical phenotyping battery. Diagnostic rates may be as high as $88 \%$ when ASD is directly evaluated as part of the study assessment process [13].

Literature on DYRK1A haploinsufficiency supports its association with ASD risk and suggests a complex phenotype that includes distinct dysmorphology as well as cognitive, neurological, and medical impairments. However, studies to date have only reported categorical descriptions of phenotype, which note the presence or absence of a common phenotype, such as ASD or no ASD. Quantitative assessments of ASD-related features in large cohorts and in relation to other ASD cohorts have not been examined. While previously published reports have noted an emerging phenotypic profile, variability in clinical presentation remains. Measurable data on medical, developmental, and behavioral characteristics are needed to better understand small variations in phenotype between individuals. Furthermore, varied clinical presentations of individuals with DYRK1A mutations have yet to be examined in the context of their familial phenotypic profile as a measure of remaining genetic background. This approach has been applied to other developmental disorders and to CNVs associated with ASD, but has yet to be applied to disruptive SNVs associated with ASD [25-28].

The aim of the proposed study was to examine a large cohort of cases with DYRK1A mutations, provide a summary of phenotype, and compare recurrent medical and behavioral features to (1) large idiopathic ASD samples and (2) a cohort with disruptive mutations to a different ASD-associated gene, CHD8. Alongside DYRK1A, CHD 8 is one of the most recurrent genes with disruptive SNVs implicated in ASD and provides a comparison group ascertained in the same way as the cases with DYRK1A mutations in this sample $[6,8]$. Detection of phenotypic differences between these two groups could inform understanding of different biological profiles of ASD and illuminate key features unique to each disrupted gene. This study also explored the contribution of genetic background to phenotypic variability among individuals with disruptive DYRK1A mutations.

\section{Methods \\ Participants \\ DYRK1A sample}

Participants included 42 individuals with de novo, disruptive, pathogenic SNVs (nonsense, splice site, frameshift, and missense mutations) at the DYRK1A gene. (Fig. 1; see Additional files 1 and 2 for full variant information). The sample includes 10 individuals assessed as part of an ongoing study at the University of Washington (UW), including 7 new cases identified through clinical genetic testing and 3 previously published cases recruited from the Simons Simplex Collection (see below). In addition to the 3 previously published cases studied at UW, 32 other previously published cases with disruptive SNVs were included in the sample. All subjects were identified via clinical exome sequencing, or exome or targeted sequencing of research cohorts ascertained for a diagnosis of ASD or ID.

Those seen at UW (UW-SNV group; $n=9$ de novo and $n=1$ non-maternal; see Table 1 for variant information [29]) completed standardized behavioral measures and medical evaluations by clinicians naïve to gene group membership as part of a study evaluating individuals ages four and older with ASD-associated, disruptive mutations. Biological parents of the participants were also characterized.

Thirty-two previously published cases of DYRK1A disruptive SNVs (Pub-SNV group) included 31 de novo 


\section{a}

Human DYRK1A gene locus
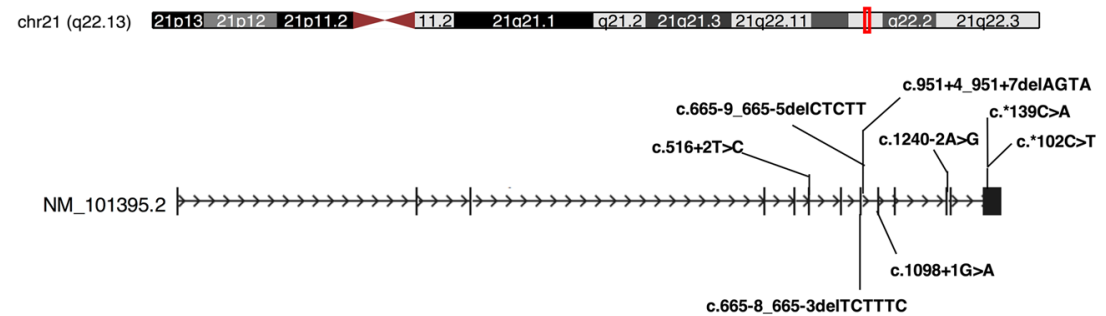

b

c.665-8_665-3deITCTTTC

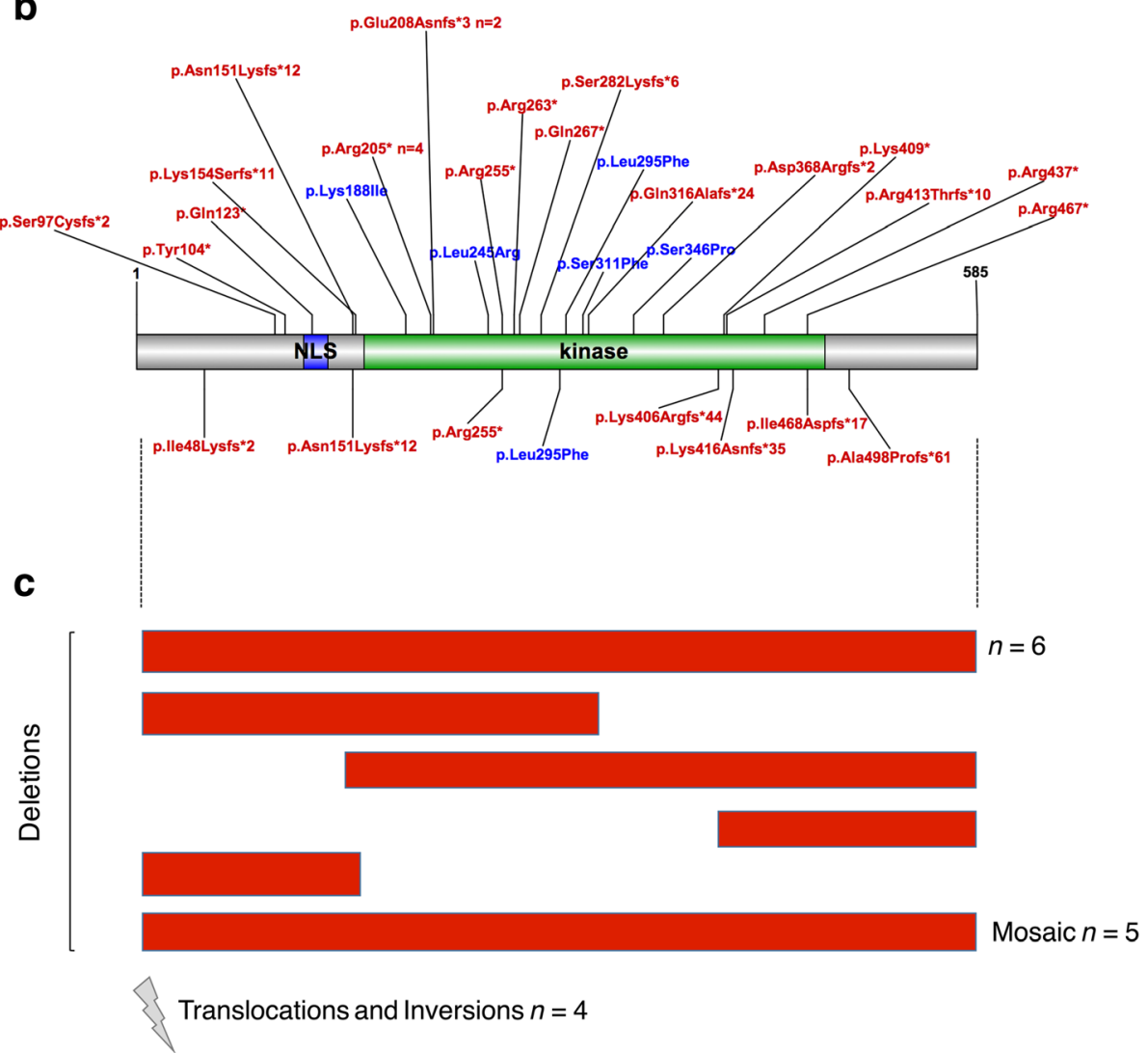

Fig. 1 Summary of DYRK1A gene variants. Schematic depicting the locations of disruptive variants (truncating, missense, and splice site mutations), copy number variations, and chromosomal rearrangements affecting DYRK1A. The ideogram of human chromosome 21 and isoform NM101395.2 coding sequence was obtained from the UCSC genome browser [54]. a NM101395.2 coding sequence with eight reported splice site mutations (presented in HGVS cDNA notation). Mutations below the sequence are UW-SNV participants, above are Pub-SNV mutation cases. b The DYRK1A protein (NP_567824.1) with truncating (red) and missense (blue) mutations (presented in HGVS notation). Mutations below the protein are UW-SNV cases, above are Pub-SNV mutation cases. c Copy number deletions and chromosomal rearrangements, including six deletions of entire gene, four partial deletions, five mosaic deletions, and four translocations/inversions (lightning bolt)

cases and 1 non-maternal case $[13,15,21,22,30-32]$ with available medical history, physical features, and diagnoses.

Additionally, the phenotype of 19 previously published cases of de novo DYRK1A chromosomal rearrangements (Pub-CHR group), including microdeletions and translocations, was described and compared to those with disruptive SNVs [21, 24, 33-39]. See Table 2 for participant characteristics for the 61 total DYRK1A sample participants.

\section{Comparison samples}

Secondary data from an idiopathic subset of the Simons Simplex Collection (SSC), a large sample ascertained for ASD, were compared to the DYRK1A sample. The SSC was a cohort of 2446 simplex families including a single proband with ASD age 4-18, unaffected biological parents, and any unaffected siblings [40]. Probands were included in the idiopathic subset $(n=1981)$ if they had no known disruptive SNVs or deleterious CNVs, as determined by sequencing efforts by Sanders and colleagues 
Table 1 DYRK1A variant information for UW-SNV mutation patients

\begin{tabular}{llllll}
\hline Patient & Position & Type of mutation & cDNA & Protein & Inheritance \\
\hline 1 & $21: 38865466$ & Splice site & c.1098+1G>A & - & De novo \\
2 & $21: 38845116$ & Frameshift & c.143_144delTA & p.lle48Lysfs*2 & De novo \\
3 & $21: 38877833$ & Frameshift & c.1491delC & p.Ala498Profs*61 & De novo \\
4 & $21: 38868533$ & Frameshift & c.1217_1220delAGAA & p.Lys406Argfs*44 De novo \\
5 & $21: 38862575$ & Nonsense & c.763C>T & p.Arg255* & De novo \\
6 & $21: 38877746$ & Frameshift & c.1401delAinsGG & p.lle468Aspfs*17 & De novo \\
7 & $21: 38853064$ & Frameshift & c.452dupA & p.Asn151Lysfs*12 & Not maternal \\
8 & $21: 38862695$ & Missense & c.883C>T & p.Leu295Phe & De novo \\
9 & $21: 38862463$ & Splice site & c.665-8_665-3delTCTTC & - & De novo \\
10 & $21: 38877590$ & Frameshift & c.1248delA & p.Lys416Asnfs*35 & De novo
\end{tabular}

Variant information for UW-SNV patients using NCBI reference sequence for DYRK1A isoform NM_101395.2, GRCh37 (hg19) build version (Ensembl id: ENST00000338785). This isoform was selected because it was the highest expressing isoform in human tissues in the GTEx database [https://gtexportal.org/home/ gene/DYRK1A]) [53]. Patients 1-3 were first identified through the Simons Simplex Collection, patients 4-10 underwent clinical genetic testing prior to research participation. CDNA and protein (NP_567824.1) annotation follows HGVS guidelines

in 2015 , and not based on any phenotypic or behavioral profile [8]. In order to account for high rates of ID seen in the DYRK1A haploinsufficiency, a subset of the idiopathic group with full-scale IQ below $70(n=487)$ served as an additional comparison group. Also, a randomly selected age- and gender-matched subset $(n=10)$ of the full idiopathic sample was compared to the subset of DYRK1A cases assessed quantitatively at the UW. As part of the SSC, probands were assessed on measures of neurocognitive functioning, social communication behaviors, motor skills, physical features (e.g., head circumference), and medical history (measures described below).

Twelve individuals with disruptive SNVs at a different high-confidence ASD risk gene, CHD8 (chromodomain helicase-DNA-binding protein 8), participating in the same UW characterization study and assessed by clinicians naïve to implicated gene disruption, served as a comparison cohort matched on ascertainment approach.

\section{Measures \\ Categorical assessment of diagnostic history and developmental characteristics}

Psychiatric and medical history, developmental milestones, and physiological characteristics were gathered from Pub-
SNV and Pub-CHR cases. In addition to published data, supplemental case reports detailing medical history and developmental trajectory were reviewed when available.

For UW-SNV study participants, a structured caregiver interview, adapted from the SSC, was administered to gather information about developmental, psychiatric, and medical history. When caregiver-endorsed diagnoses required additional clarification, medical records were reviewed for confirmation. Using all available information, psychiatric diagnoses were either confirmed or newly diagnosed by a licensed clinical psychologist using the Diagnostic and Statistical Manual of Mental Disorders, 5th Edition (DSM-5) [16]. For research purposes, subjects were given an ASD diagnosis based on clinician observation and parent interview using standardized instruments (gold-standard assessment tools described below). A diagnosis of intellectual disability was given when a subject displayed childhood onset of deficits in both cognitive and adaptive functioning. When subjects were under the age of 5 and had failed to reach cognitive developmental milestones at the time of assessment, a diagnosis of Global Developmental Delay was given. A physical and dysmorphology exam was conducted by a licensed medical geneticist.

Table 2 Demographics

\begin{tabular}{|c|c|c|c|c|c|c|}
\hline & \multicolumn{3}{|l|}{ DYRK1A sample } & \multirow{2}{*}{\multicolumn{2}{|c|}{$\begin{array}{l}\text { SSC idiopathic sample } \\
\text { No disruptive SNVs or deleterious CNVs }\end{array}$}} & \multirow{3}{*}{$\begin{array}{l}\text { CHD8 sample } \\
\text { Disruptive SNVs }\end{array}$} \\
\hline & \multicolumn{2}{|l|}{ Disruptive SNVs } & \multirow{2}{*}{$\begin{array}{l}\text { CHR } \\
\text { Pub-CHR }\end{array}$} & & & \\
\hline & Pub-SNV & UW-SNV & & Total sample & $\mathrm{IQ}<70$ & \\
\hline Total N (male) & $32(22)$ & $10(4)$ & $19(9)$ & $1981(1705)$ & $487(407)$ & $12(9)$ \\
\hline Mean age in months (SD) & $124.12(128.85)$ & $108.40(69.12)$ & $102.22(88.06)$ & $107.66(42.34)$ & $114.00(44.00)$ & $148.08(64.56)$ \\
\hline
\end{tabular}

Participant demographics. SNV single nucleotide variant, Pub-SNV published disruptive SNV cases, UW-SNV UW study cases with disruptive SNVs, Pub-CHR published chromosomal rearrangement, CNV copy number variant. Note that there are three overlapping individuals in the Pub-SNV and UW-SNV groups ascertained from the Simons Simplex Collection. DYRK1A sample significantly differed from idiopathic ASD samples (total and IQ $<70)$ in gender ratio, $X^{2}(1$, $n=2042)=66.88, p<0.001$ and $x^{2}(1, n=548)=36.25, p<0.001$, respectively. Samples did not significantly differ in age, $p>0.05$. No significant differences in age or gender for DYRK1A and CHD8 samples 
Quantitative assessment of DYRK1A UW-SNV $(n=10)$ and

CHD8 $(n=12)$

Head circumference

Occipital frontal head circumference was measured and standardized values calculated using a normative population reference [41].

\section{Cognitive functioning}

Full-scale IQ was assessed in probands and unaffected parents. Probands ages 4 years, 0 months to 17 years, 11 months were administered the Differential Abilities Scales, 2nd Edition [42]. Probands 18 and older, as well as unaffected parents, were administered the Wechsler Abbreviated Scales of Intelligence [43]. For all assessments, IQ scores were generated using deviation (standard; mean $=100, \mathrm{SD}=15$ ) or ratio scores (mental age equivalent/chronological age $\times 100)$. Ratio scores were derived using age equivalence values if standard scores were not possible to calculate due to subject's level of functioning.

\section{Adaptive functioning}

Caregivers were administered the Vineland Adaptive Behavior Scales, 2nd edition (VABS-2) to measure adaptive functioning across communication, daily living skills, and social domains [44].

\section{ASD-specific assessment}

Research-reliable clinicians administered the appropriate module of the Autism Diagnostic Observation Schedule, 2nd Edition (ADOS-2; [45, 46]) and Autism Diagnostic Interview-Revised (ADI-R; [47]). ADOS calibrated severity scores, and items regarding age of first words and age of first steps from the ADI-R were used in analyses. The total $T$ score from the Social Responsiveness Scale (SRS2 ; [48]) was used to quantify ASD-associated symptoms in all UW-SNV family members.

\section{Analytic approach \\ Categorical variables}

Fisher's exact tests were used to compare frequencies of features commonly found for DYRK1A mutations across disruptive SNV (Pub-SNV and UW-SNV) and chromosomal rearrangement (Pub-CHR) groups. These features included intellectual disability, speech delay (defined as first words after 24 months of age), motor deficits (e.g., delayed walking, poor coordination, abnormal gait), ASD-related deficits (e.g., ASD diagnosis, stereotypic behaviors, anxious behaviors), feeding difficulties, seizures, vision abnormalities, and microcephaly. The frequency of most common features (defined as present in $75 \%$ or more cases) was compared across DYRK1A and idiopathic ASD groups (total idiopathic sample and subset with IQ below 70). Only characteristics specifically noted in case reports were included in analyses; if a phenotypic characteristic was not reported, it was treated as missing for that individual. Total frequencies reflect cases with a reported presence or absence of a given characteristic.

\section{Quantitative variables}

UW-SNV DYRK1A participants were compared to (1) a randomly subsampled age and gender-matched subset of the SSC idiopathic sample and (2) a cohort with disruptive CHD8 mutations on domains of functioning assessed quantitatively, including head circumference, IQ, adaptive functioning, ASD severity (ADOS calibrated severity score), age of first words (ADI-R), and age of first independent steps (ADI-R). Independent sample $t$ tests were used to compare the DYRK1A, idiopathic, and CHD 8 groups, using the Bonferroni adjustment for multiple comparisons $(p<0.002)$.

Nonparametric Wilcoxon signed rank tests were used to compare parental and proband phenotype for UWSNV participants in head circumference, IQ, and ASD symptoms (SRS). Gene "effect size," measured as the difference between parental and proband phenotype, was calculated as follows:

$$
\text { Effect size }=\frac{\text { Proband mean-Unaffected biparental mean }}{\text { Unaffected biparental standard deviation }}
$$

When both maternal and paternal data were available, biparental means were calculated as the average of maternal and paternal scores. If only one parent's data was available, that parent's score was used instead of a biparental mean.

\section{Results}

\section{Clinical phenotype of DYRK1A}

There were no significant differences between disruptive SNV (Pub-SNV and UW-SNV) and chromosomal rearrangement (Pub-CHR) groups on frequency of phenotypic characteristics (Table 3 ). Language delay was noted for $61 / 61$ (100\%); 21 individuals were nonverbal at the time of their evaluation. Intellectual disability and/or Global Developmental Delay (depending on age) were reported in 60/61 (98\%) cases. The presence of motor difficulties, including delayed walking, abnormal gait, and poor coordination, was noted for 52/53 (98\%). A common abnormal gait was observed across UW-SNV participants, specifically a lilting gait with a forward lean to the upper body, arms bent and held tight against the body, and hands splayed. Feeding difficulties in infancy, including poor suck, were observed in 51/54 (94\%) of those with reports of feeding abilities in early development. Microcephaly, defined as head circumference two or more standard deviations below the mean for age, either primary (present throughout development) or 
Table 3 Phenotypic characteristics of DYRK1A

\begin{tabular}{|c|c|c|c|c|c|c|c|c|c|c|}
\hline \multirow[b]{2}{*}{ Phenotypic characteristic } & \multicolumn{3}{|c|}{ Pub-SNV and UW-SNV $(n=42)$} & \multicolumn{3}{|c|}{ Pub-CHR $(n=19)$} & \multicolumn{3}{|c|}{ Total $(n=61)$} & \multirow[b]{2}{*}{ Sig (Fisher's exact tests) } \\
\hline & $\bar{N}$ & Total & $\%$ & $\bar{N}$ & Total & $\%$ & $\bar{N}$ & Total & $\%$ & \\
\hline Intellectual disability or Global Developmental Delay & 41 & 42 & 98 & 19 & 19 & 100 & 60 & 61 & 98 & NS \\
\hline Speech delay & 42 & 42 & 100 & 19 & 19 & 100 & 61 & 61 & 100 & NS \\
\hline Motor difficulties & 38 & 38 & 100 & 14 & 15 & 93 & 52 & 53 & 98 & NS \\
\hline Microcephaly & 39 & 42 & 93 & 19 & 19 & 100 & 58 & 61 & 95 & NS \\
\hline Feeding difficulties & 37 & 40 & 93 & 14 & 14 & 100 & 51 & 54 & 94 & NS \\
\hline Vision abnormalities & 26 & 33 & 79 & 8 & 9 & 89 & 34 & 42 & 81 & NS \\
\hline Seizures & 26 & 39 & 67 & 16 & 19 & 84 & 42 & 58 & 72 & NS \\
\hline ASD diagnosis & 16 & 35 & 46 & 2 & 7 & 29 & 18 & 42 & 43 & NS \\
\hline Stereotyped behaviors & 22 & 36 & 61 & 4 & 9 & 44 & 26 & 45 & 58 & NS \\
\hline Anxious behaviors & 11 & 36 & 31 & 1 & 8 & 13 & 12 & 44 & 27 & NS \\
\hline Hyperactive behaviors & 10 & 35 & 29 & 4 & 8 & 50 & 14 & 43 & 33 & NS \\
\hline Behavioral differences & 35 & 42 & 83 & 7 & 19 & 37 & 42 & 61 & 69 & NS \\
\hline 6+ symptoms including ASD & 25 & 42 & 60 & 7 & 19 & 37 & 32 & 61 & 52 & NS \\
\hline 6+ symptoms including broader behavioral difficulties & 32 & 42 & 76 & 10 & 19 & 53 & 42 & 61 & 69 & NS \\
\hline
\end{tabular}

Frequency of phenotypic features in cases with disruptive SNVs (Pub-SNV and UW-SNV) to DYRK1A, published chromosomal rearrangements (Pub-CHR) to DYRK1A, and total combined cases. Totals reflect those with complete data. Groups did not significantly differ in gender ratio (Fisher's exact test) or age (independent sample $t$ test), $p>0.05$. Fisher's exact tests used for group comparisons, Sig significance, NS not significant

acquired at a later age, was reported in $58 / 61$ cases (95\%). Vision abnormalities were identified in $34 / 42$ (81\%) cases, including impairments such as strabismus, astigmatism, optic nerve dysfunction, and corneal clouding. Febrile and non-febrile seizures were reported in $42 / 58$ cases $(72 \%)$.

Diagnoses of ASD were reported in 18/42 cases (43\%), suggesting elevated risk well above the general population percentage of $1.5 \%$ [49]. The frequency increased to $42 / 61$ cases $(69 \%)$ when broadening the criteria to include ASD-related behaviors without a formal diagnosis, such as stereotypic behaviors (e.g., complex motor mannerisms, repetitive and self-stimulatory behaviors), limited eye contact (reported in those without known severe vision impairments), inappropriate laughter, and limited social engagement. Anxious behaviors were reported in 12/44 cases (27\%), and hyperactivity was reported in 14/43 cases (33\%). Seven of the ten UW-SNV cases were confirmed to have ASD; three who did not meet diagnostic criteria presented with notable stereotypies and socially anxious behaviors.

Co-occurrence of the seven most common phenotypic features (reported in $75 \%$ or more of cases) was evaluated: microcephaly, intellectual impairment, speech delay, motor difficulties, feeding difficulties, vision abnormalities, and ASD. Fifty-two percent of the total DYRK1A sample (32/61) possessed six or more features. Sixty-nine percent $(42 / 61)$ presented with six or more features when the ASD category was broadened to include other behavioral difficulties, including stereotypic, anxious, and hyperactive behaviors.
Facial dysmorphisms were reported in 50/51 (98\%) previously published cases (excluding UW-SNV cases who were previously published, $n=3$ ). Similar dysmorphic facial features were observed in eight UW-SNV cases who participated in a standardized medical exam (five new cases, three previously published), including deep-set eyes with a hooded appearance, slightly upslanting palpebral fissures, bitemporal narrowing, prominent brow with high anterior hairline, tubularshaped nose, prominent nasal bridge, retrognathic jaw, and small chin (Fig. 2a). Additionally, prominent, lowset, or malformed ears were also reported across cases; 4/8 UW-SNV cases presented with thick, overfolded ear helices (Fig. 2b). Foot anomalies were also noted across patients, including toe syndactyly (webbing of the toes), arachnodactyly, crooked toes, and proximal placement of the first toe (Fig. 2c). Observed commonalities in facial, ear, and foot characteristics in UW-SNV cases were consistent with reports of previously published cases. In the larger sample, spine or chest abnormalities, including pectus excavatum and scoliosis, were reported in 13/ 25 cases with documented skeletal observations.

\section{Phenotypic comparisons of DYRK1A to idiopathic ASD}

Rates of microcephaly, intellectual disability, speech delay, motor difficulties, vision impairments, and feeding difficulties were significantly higher in the total DYRK1A group (Pub-SNV, UW-SNV, and Pub-CHR combined) relative both to the full idiopathic SSC comparison cohort and the subset with IQ below 70 (Table 4; Fig. 3). 
a

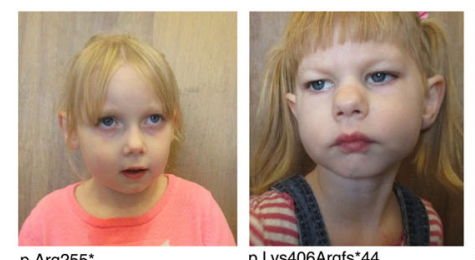
p.Arg255*

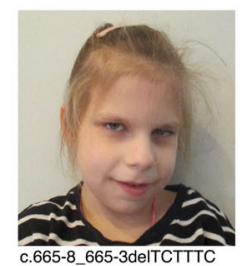

b

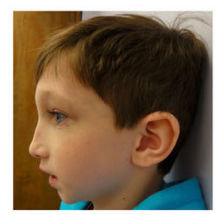

p.Ala498Profs $* 61$

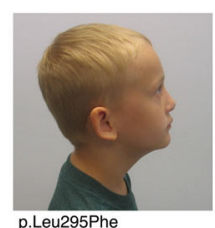

p.Leu295Phe

C

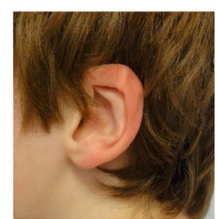

p.lle48Lysfs*2

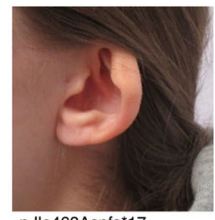

p. lle468Aspfs ${ }^{\star} 17$

d

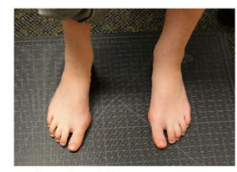

p.lle48Lysfs*2

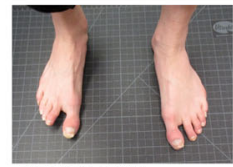

p.lle468Aspfs*17

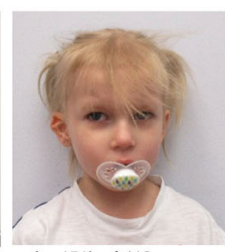

p.Asn151Lysfs ${ }^{\star} 12$
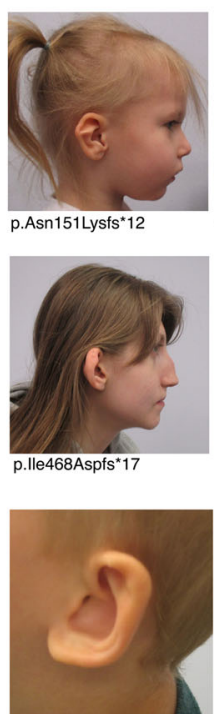

p.Leu295Phe

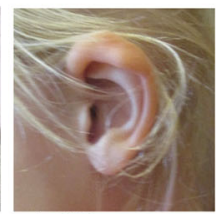

p.Arg255*

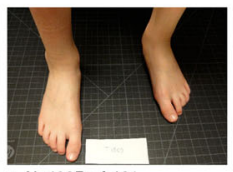

p.Ala498Profs ${ }^{*} 61$

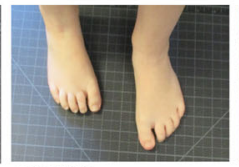

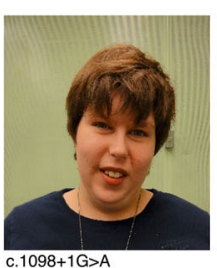

c. $1098+1 \mathrm{G}>\mathrm{A}$

p.Asn151Lysfs*12

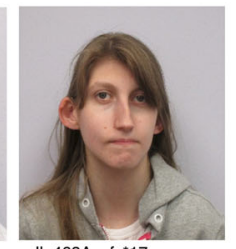

p.lle468Aspfs 17
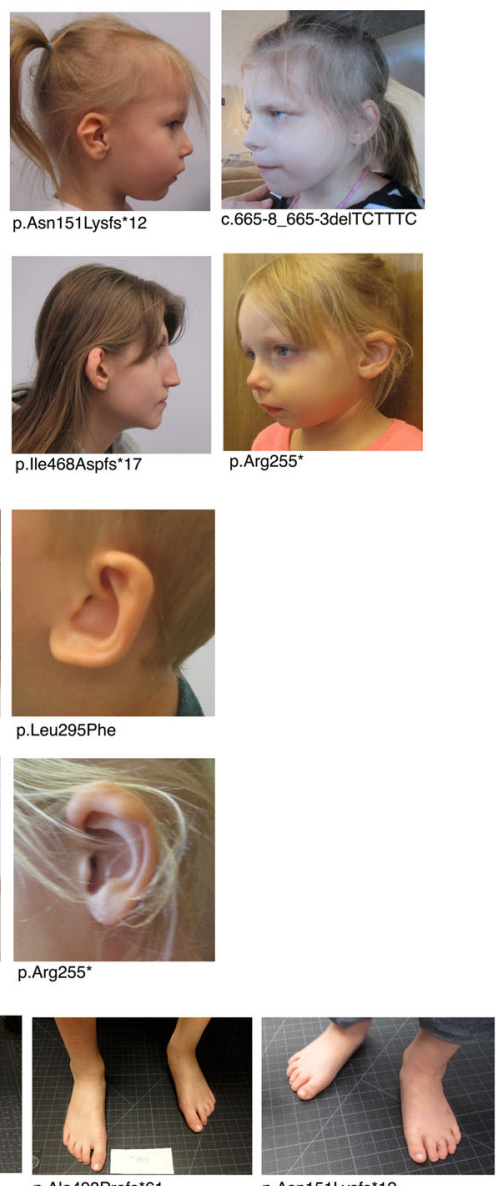

p.Asn151Lysfs*12

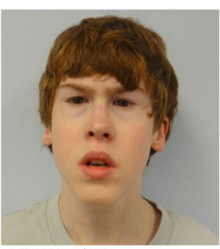

p.lle48Lysfs*2

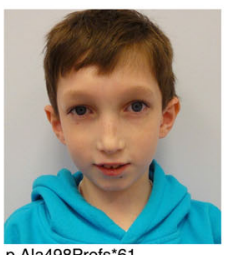

p.Ala498Profs*61

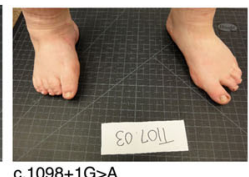

p.Lys416Asnfs"35
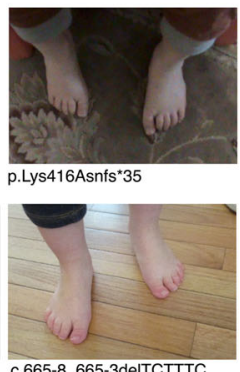

Fig. 2 (See legend on next page.) 
(See figure on previous page.)

Fig. 2 Common dysmorphic features in UW-SNV patients with DYRK1A haploinsufficiency. a Facial features of eight UW-SNV patients with DYRK1A haploinsufficiency. Note common features across patients, including prominent brow with high anterior hairline, slightly upslanted palpebral fissures, retrognathic jaw, deep-set eyes with a hooded appearance, bitemporal narrowing, high nasal bridge with tubular-shaped, broad-tipped nose, and protruding ears. b Profiles of six UW-SNV patients. Note prominent brows with high anterior hairlines as well as low-set, posteriorly rotated ears in a subset of patients. c Ear abnormalities in four UW-SNV patients, including post-rotated and protruding ears with protruding thick and overfolded helices (i.e., outer fold of the ear). $\mathbf{d}$ Foot abnormalities in eight UW-SNV patients. Common features include proximal placement of the first toe, crooked toes, and syndactyly of the second and third toes. Frameshift, nonsense, and missense cases identified by HGVS protein notation; cases with splice site variants identified by HGVS cDNA notation

The majority of the DYRK1A group (79\%) displayed five or more of these phenotypic features in combination. The percentages and group differences remain in the subset of individuals with DYRK1A mutations ascertained for an ASD diagnosis ( $n=18$, Fig. 3). Cooccurrence of five or more features increased to $89 \%$ in those with $D Y R K 1 A$ mutations ascertained for ASD.

\section{Quantitative phenotype of DYRK1A DYRK1A vs idiopathic ASD}

Independent sample $t$ tests revealed significant differences between UW-SNV and a matched idiopathic group on measures of head circumference, cognitive ability, and adaptive functioning (Table 5; Fig. 4). The UW-SNV group had significantly smaller head circumference $(p<0.001)$, significantly lower full-scale IQ $(p=0.002)$, and significantly lower adaptive abilities $(p=0.001)$ compared to the idiopathic group. There were no differences between groups on autism symptom severity (ADOS calibrated severity score), age of first words, or age of first independent steps. Outliers across the six phenotypic features of interest represented different UW-SNV individuals, highlighting the variability that is observed when exploring the phenotype quantitatively.

\section{DYRK1A vs CHD8}

No significant differences were found between $C H D 8$ and idiopathic groups. However, UW-SNV and CHD8 groups differed significantly in head circumference $(p<0.001)$, such that DYRK1A cases had significantly smaller head size than CHD8 cases. IQ, adaptive functioning, autism symptom severity, age of first words, and age of first steps were similar across groups (Table 5; Fig. 4).

\section{Contribution of genetic background}

When comparing parental and proband head circumference $Z$ score, the presence of a DYRK1A mutation accounted for a 2.93 SD decrease in head size for probands. Wilcoxon signed rank tests showed that both mothers and fathers exhibited significantly larger head size, controlling for age and gender, compared to their affected child $(Z=-2.67, p=0.008$ and $Z=-2.20$, $p=0.028$, respectively (Fig. 5a)). When comparing ASDrelated symptoms, DYRK1A accounted for a $5.51 \mathrm{SD}$ increase in SRS total $T$ score (more symptoms). Wilcoxon signed rank tests showed that both mothers and fathers display significantly lower SRS scores compared to their affected child $(Z=-3.62, p<0.001$ and $Z=-3.41$, $p=0.001$, respectively (Fig. 5b)). On measures of fullscale IQ, DYRK1A accounted for a 6.09 SD decrease in IQ for probands compared to biparental IQ. Wilcoxon signed rank tests showed that both mothers and fathers display significantly higher IQ compared to their affected child $(Z=-2.67, p=0.008$ and $Z=-2.20, p=0.028$, respectively (Fig. 5c)).

\section{Discussion}

This study of the DYRK1A haploinsufficiency phenotype, compiling previously published and newly identified cases, confirms a phenotype characterized by microcephaly, intellectual disability, speech delay, motor difficulties, feeding difficulties, and vision abnormalities. A common facial gestalt included deep-set eyes with a hooded appearance, slightly upslanted palpebral fissures, tubular-shaped nose with pronounced broad tip, high nasal bridge, prominent brow with high anterior hairline, retrognathic jaw, and small chin. Dysmorphic feet, including proximal placement of the first toe, syndactyly of the second and third toe, and unusually long and/or crooked toes, and protruding, post-rotated ears with overfolded, thick helices were also commonly observed. Those with de novo disruptive SNVs and chromosomal rearrangements did not differ in clinical features.

Of those case studies where ASD was mentioned and/ or evaluated, $43 \%$ of probands received an ASD diagnosis. Among 15 cases who received gold-standard ASD assessment, rates increased to $73 \%$. Additionally, features common to ASD, such as stereotypic and anxious behaviors, were noted in many cases where reference to an ASD diagnosis was absent. This suggests rates of ASD in $D Y R K 1 A$ cohorts may be higher in reality than reported in the total sample of $D Y R K 1 A$ cases published to date.

There are several reasons for the potential underestimated prevalence rate of ASD among published DYRK1A cases. First, most previously published cases relied on medical records, which varied greatly in the 


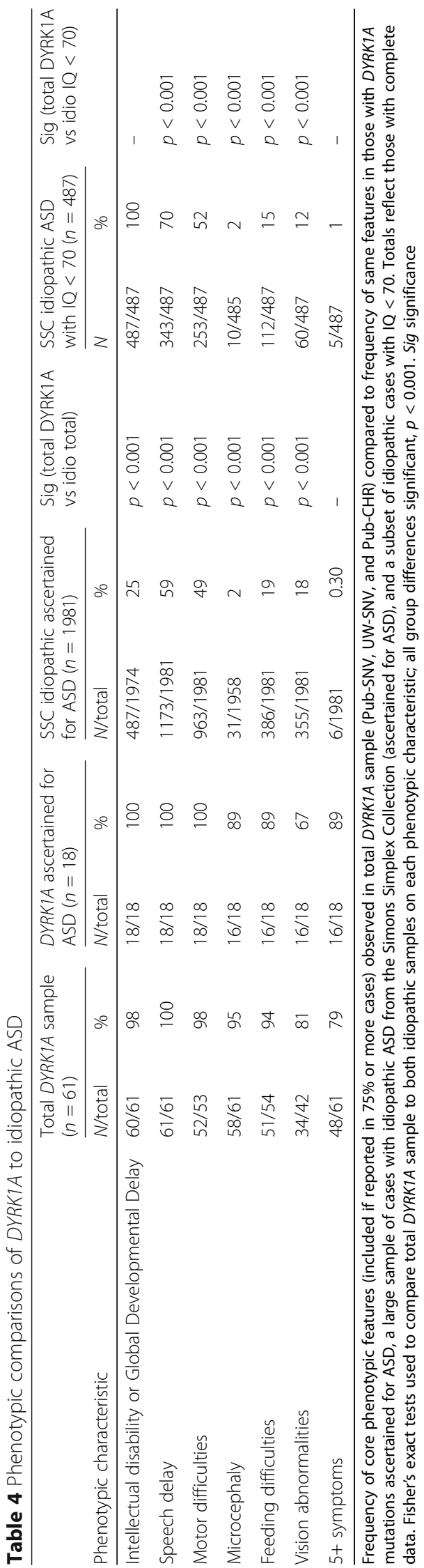




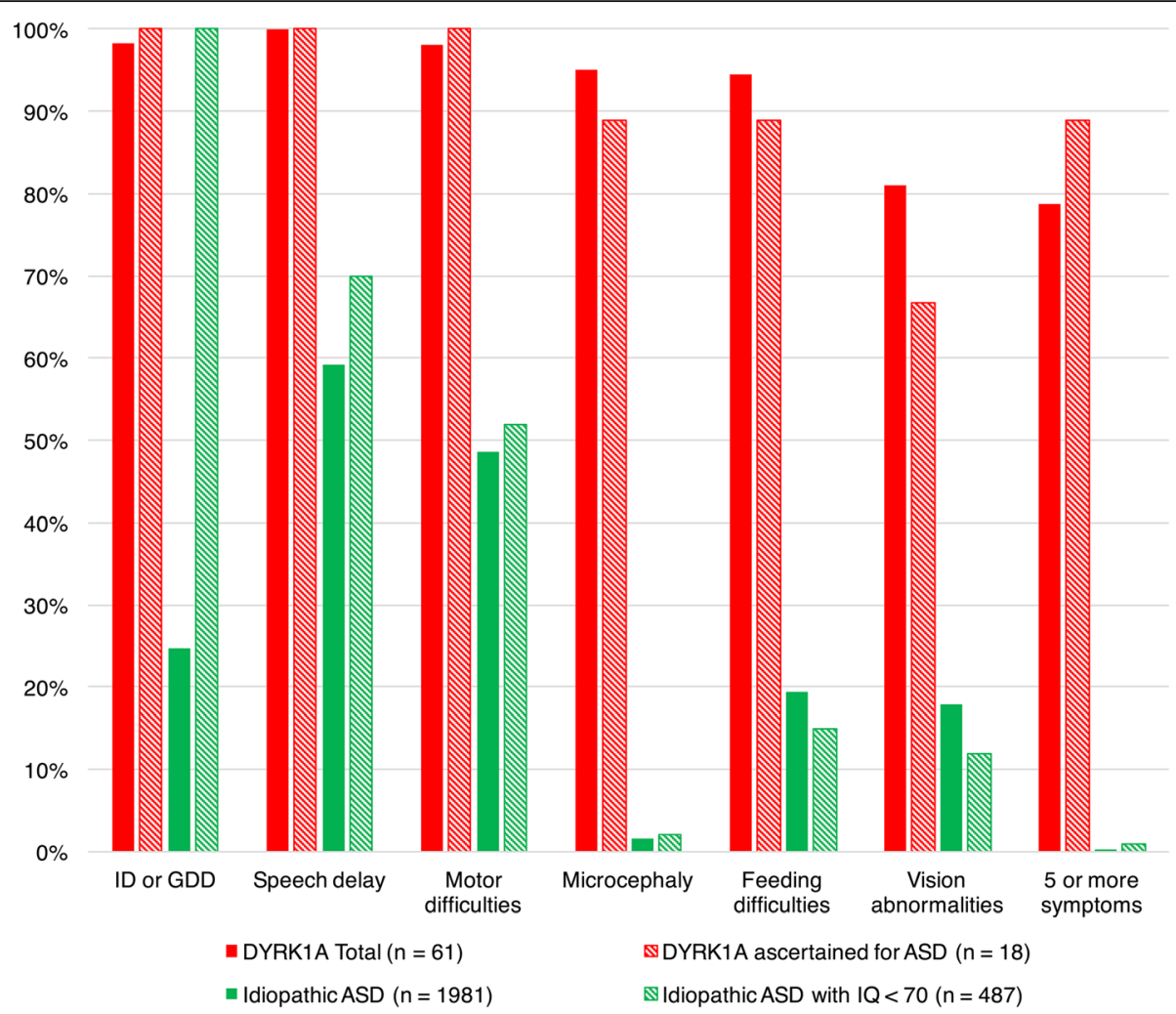

Fig. 3 Phenotypic features in total DYRK1A sample, DYRK1A sample ascertained for ASD, and idiopathic ASD samples. Bar graph presented frequencies of core phenotypic features observed in 75\% or more of DYRK1A patients. Total DYRK1A sample (Pub-SNV, UW-SNV, Pub-CHR) and DYRK1A sample ascertained for ASD were compared to frequencies of features in idiopathic ASD samples (total and IQ $<70)$ using Fisher's exact tests $(p<0.001$ )

detail provided and discussion of comprehensiveness of prior evaluations; as such, it is unknown whether ASD was either evaluated and ruled out, or not evaluated at all. Second, it can be difficult to tease apart symptoms of ASD from those of intellectual disability and speech impairments without specialized training and experience with differential diagnosis within developmental disabilities, particularly in children with complex medical histories. Additionally, establishing an ASD diagnosis may not be the most pressing concern for families (and perhaps providers) given the array of impairments and medical conditions that often accompany children with a $D Y R K 1 A$ mutation. As DYRK1A haploinsufficiency continues to be explored within ASD risk, these factors need to be considered when determining rates in this population.

In an effort to situate the DYRK1A phenotype in the context of ASD, we found the DYRK1A group (Pub-

Table 5 Quantitative phenotype and group differences between DYRK1A, idiopathic, and CHD8 groups

\begin{tabular}{|c|c|c|c|c|c|c|c|c|c|c|c|c|c|c|c|}
\hline \multirow[b]{2}{*}{ Phenotypic characteristic } & \multicolumn{3}{|c|}{$\begin{array}{l}\text { DYRK1A } \\
\text { (UW-SNV) }\end{array}$} & \multicolumn{3}{|c|}{$\begin{array}{l}\text { SSC idiopathic } \\
\text { subset }\end{array}$} & \multicolumn{3}{|l|}{ CHD8 } & \multicolumn{2}{|c|}{$\begin{array}{l}\text { t statistics DYRK1A } \\
\text { vs SSC }\end{array}$} & \multicolumn{2}{|c|}{$\begin{array}{l}\text { t statistics DYRK1A } \\
\text { vs CHD8 } \\
\end{array}$} & \multicolumn{2}{|c|}{$\begin{array}{l}\text { t statistics CHD8 } \\
\text { vs SSC } \\
\end{array}$} \\
\hline & Mean & SD & $N$ & Mean & SD & $N$ & Mean & SD & $N$ & $t$ & $d$ & $t$ & $d$ & $t$ & $d$ \\
\hline Head circumference $Z$ score & -3.62 & 2.17 & 10 & 1.21 & 0.39 & 10 & 1.76 & 2.9 & 11 & $6.94^{*}$ & 3.10 & $6.49^{* *}$ & 2.84 & NS & - \\
\hline Full-scale IQ & 45.30 & 18.14 & 10 & 81.20 & 26.12 & 10 & 60.91 & 27.39 & 11 & $3.57^{*}$ & 1.60 & NS & - & NS & - \\
\hline Overall adaptive functioning & 54.80 & 9.04 & 10 & 70.50 & 9.40 & 10 & 65.00 & 18.49 & 12 & $3.81^{*}$ & 1.70 & NS & - & NS & - \\
\hline Autism severity (ADOS CSS) & 6.50 & 2.80 & 10 & 7.70 & 1.25 & 10 & 8.18 & 1.78 & 11 & NS & - & NS & - & NS & - \\
\hline Age walked unaided & 19.70 & 5.64 & 10 & 13.22 & 2.59 & 10 & 18.50 & 4.38 & 12 & NS & - & NS & - & NS & - \\
\hline Age of first single words & 45.14 & 22.82 & 7 & 17.50 & 7.92 & 10 & 25.17 & 28.01 & 12 & NS & - & NS & - & NS & - \\
\hline
\end{tabular}

Descriptives for phenotypic variables commonly impaired in DYRK1A haploinsufficiency for three groups: DYRK1A ( $n=10)$, SSC idiopathic subset randomly samples and matched on age and gender $(n=10)$, and CHD8 $(n=12)$. Group comparisons using independent sample $t$ tests between $(1)$ DYRK1A and SSC groups, (2) DYRK1A and CHD8 groups, and (3) CHD8 and SSC groups. *Significant differences between DYRK1A and idiopathic groups, $p<0.002 ; * *$ Significant differences between DYRK1A and CHD8 groups, $p<0.001$. Independent sample $t$ and Cohen's $d$ values provided when significant, $p$ value adjusted for multiple comparisons, NS not significant 

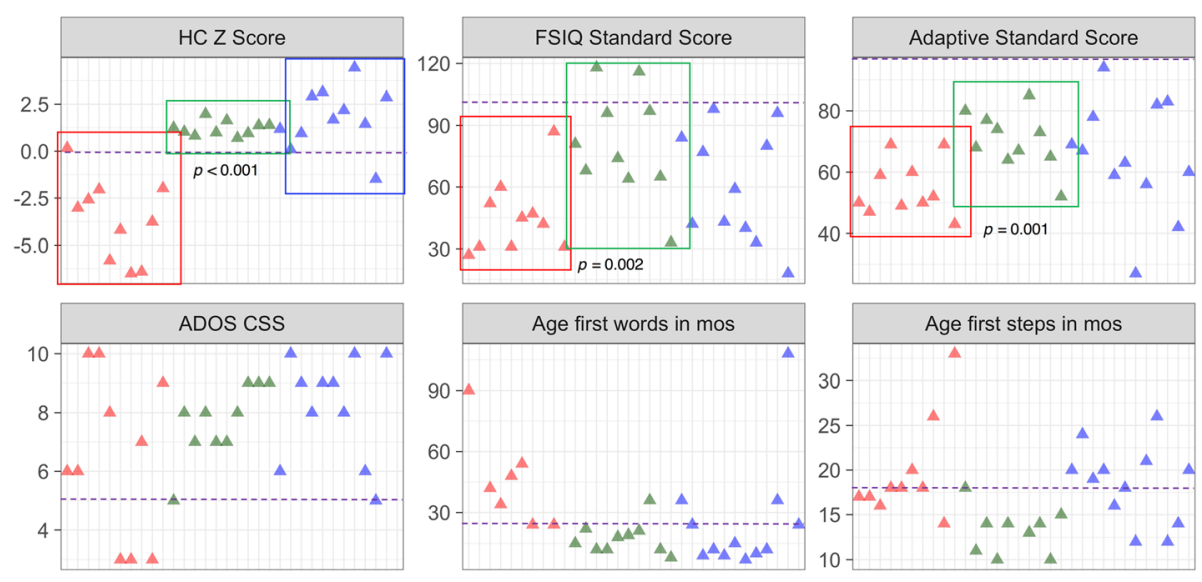

$\triangle \mathrm{DYRK} 1 \mathrm{~A} \triangle \mathrm{SSC} \triangle \mathrm{CHD8}$

Fig. 4 Quantitative phenotype of DYRK1A, idiopathic, and CHD8 samples. Scatterplots of core phenotypic features in UW-SNV DYRK1A sample, $(n=10)$, idiopathic subset matched for age and gender (randomly sample, $n=10)$, and CHD8 sample $(n=12)$. Dotted lines designate conservative averages for typical population. HC head circumference, FSIQ full-scale IQ, ADOS CSS calibrated severity score. Independent sample $t$ tests comparing DYRK1A,

idiopathic, and CHD8 groups, $p$ value adjusted for multiple comparisons

SNV, UW-SNV, and Pub-CHR groups combined) exhibited significantly higher incidence of key features compared to those with idiopathic ASD: intellectual disability, speech delay, motor difficulties, vision abnormalities, feeding difficulties, and microcephaly. Frequency of these features also significantly differed between the DYRK1A group and the comparison group with idiopathic ASD and IQ below 70. This is consistent with prior evidence that disruptive SNVs and CNVs often result in significantly more impairing comorbidities than in idiopathic ASD $[6,8]$. Notably, when those with DYRK1A mutations who were originally ascertained for an ASD diagnosis were compared to the idiopathic group (also ascertained for ASD), the profile remains the same. This provides further support that the phenotype commonly exhibited in individuals with DYRK1A disruptions and ASD is indeed distinct from idiopathic ASD. The co-occurrence of five or more of these phenotypic features in DYRK1A cases (79\% of total sample, $89 \%$ of those ascertained for ASD) provides support for further exploration of DYRK1A haploinsufficiency in an individual presenting with concerns of ASD and this combination of phenotypic features.

Prior publications of DYRK1A mutation cases have relied on categorical data to describe clinical phenotype. Our exploration of a quantitative phenotype suggested that DYRK1A haploinsufficiency is differentiable from idiopathic ASD by measures of cognition, adaptive skills, and head size and distinguishable from a different ASDassociated gene mutation, CHD8 by head size. It is possible that further phenotypic differences exist which have not been detected by current diagnostic tools given limits to the level of resolution inherent in clinical assessment. Markers relying on quantitative, brain-based measures may reveal gene-specific profiles. For instance, recent work highlights divergent information processing systems for children with 16p11.2 CNVs [50] and children with an early-emerging disruptive SNV [51]. Considering the intellectual disability associated with DYRK1A haploinsufficiency, a passive, noninvasive neuroimaging approach may help illuminate neuroendophenotypes that link the behavioral phenotype to the underlying neural mechanisms.

Exploring quantitative phenotype in UW-SNV participants illuminated phenotypic heterogeneity among individuals. While DYRK1A mutations significantly impact functioning in a number of domains, the severity of impairment varied among individuals. Family background may, in part, contribute to this variability. While still exploratory, variability in parental phenotype corresponded with variability observed in probands with DYRK1A haploinsufficiency. Most striking were familial patterns on measures of head circumference. Even with the range of microcephaly, probands with the smallest head sizes were related to parents with smaller head sizes compared to other parents within the UW-SNV group. Physiological characteristics are among the most highly correlated between parents and children in typically developing populations, ranging from 0.5 to $0.7[52,53]$. Our findings suggest that, even in the presence of a de novo, disruptive DYRK1A mutation, parental phenotype may still impact their affected child's presentation. Of course, secondary genetic events, embryonic or early developmental influences, and treatment must also be considered as potential factors contributing to the variability.

Our findings must be considered in the context of limitations of this study. First, information available for 


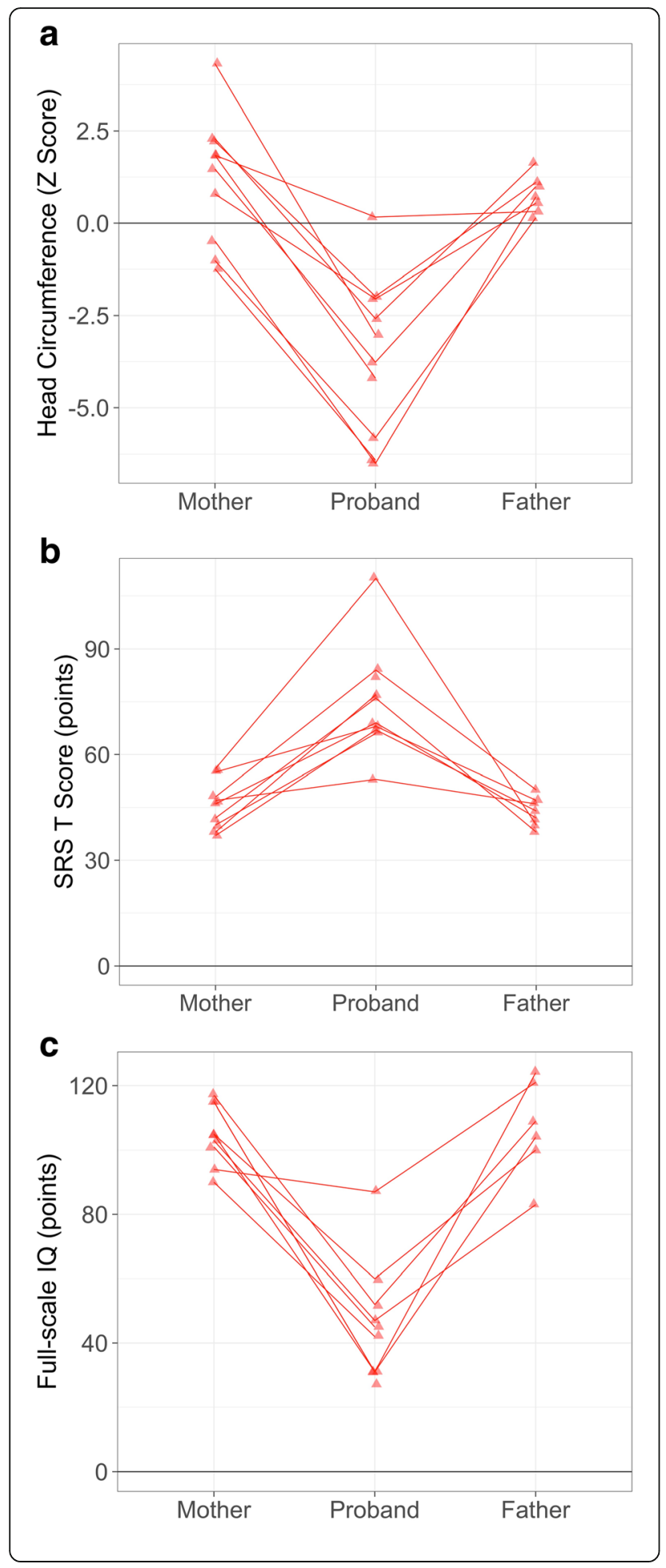

Fig. 5 Contribution of familial genetic background to head circumference, ASD symptoms, and IQ. UW-SNV cases are presented with their unaffected mothers and fathers on three phenotypic measures: a head circumference (Z score, SD), b ASD symptoms (Social Responsiveness Scale T score), and c IQ (full-scale standard score). Affected children presented with significantly more severe phenotypes compared to both unaffected mothers and fathers using Wilcoxon rank sum tests $(p<0.001)$. Variability in parental phenotype corresponds to proband variation. Probands with smaller head sizes relative to other UW-SNV cases correspond to parents who also have smaller head size and vice versa. There are similar patterns in cognition, perhaps more pronounced for fathers, such that fathers with higher IQ have probands with higher IQ relative to other DYRK1A cases. Related to social responsiveness, higher parental scores (i.e., greater social impairment) correspond to probands with greater social impairment. Also, note the apparent wider range of IQ variability for fathers $(S D=14.99)$ relative to mothers $(S D=9.42)$ and the wider range of head circumference variability for mothers $(S D=1.81)$ relative to fathers $(S D=0.52)$

previously published cases varied widely. While some case reports provided detailed record of psychiatric history, others only included medical history, which also varied in its extensiveness. Full assessment history was unknown for previously published cases, raising questions whether phenotypic features left out of a case report were previously ruled out and confirmed absent or were not assessed. These variations highlight the importance of consistency in phenotypic assessment across future DYRK1A phenotype studies to ensure comprehensive and accurate phenotyping efforts. Second, those with DYRK1A mutations who participated in the same quantitative assessment battery remain small in number. Larger sample sizes are indeed needed to better understand the quantitative phenotype of DYRK1A haploinsufficiency and potential variability between affected individuals. Also, while comparison of DYRK1A mutation cases to idiopathic ASD provides important confirmation of distinct comorbidities within ASD, it is important to acknowledge that individuals in the idiopathic group may, with future advances in our understanding of the genetics of ASD, no longer be identified as idiopathic. The idiopathic group analyzed in this study likely represents a population with fewer syndromic features than populations with ASD and other genetic events. Thus, further studies and larger samples of other ASD-associated gene mutations are needed to further distinguish how the DYRK1A haploinsufficiency phenotype differs from that of other disruptive gene events. Future studies of this population should also aim for greater specificity in phenotypic characterization in efforts to better understand DYRK1A haploinsufficiency as a unique clinical profile. Continued study of ASDassociated genes, including $D Y R K 1 A$, will allow for improved understanding of ASD subtypes and inform future approaches to personalized treatment. 


\section{Conclusions}

DYRK1A haploinsufficiency results in a clinical phenotype which includes microcephaly, intellectual impairment, the presence of vision and motor difficulties, feeding difficulties, language delays, and ASD risk. The DYRK1A profile suggests a potential subtype of ASD. Despite a consistent profile, quantitative assessment highlights heterogeneity in the severity of impairments, with parental phenotype, reflecting genetic background, as a likely contributor to that variability among individuals.

\section{Additional files}

Additional file 1: Variant information for known de novo DYRK1A mutation cases. Full variant information for previously published DYRK1A variants and patients seen at UW. Previously published cases identified by first author last name and UW cases denoted TXXX. Sheets organized by variant type: snvs/indels, cnvs, mosaic variants, and translocations/ inversions. (XLSX $17 \mathrm{~kb}$ )

Additional file 2: Variant locations via UCSC Genome Browser. Presentation of DYRK1A isoforms and variant locations for previously published and UW cases. Figure generated in UCSC Genome Browser [54]. (PDF $201 \mathrm{~kb})$

\section{Abbreviations}

ASD: Autism spectrum disorder; CHR: Chromosomal rearrangement (including CNVs, translocations, and inversions); CNV: Copy number variation; Pub-CHR: Previously published chromosomal rearrangements; PubSNV: Previously published disruptive SNVs; SNV: Single nucleotide variant (disruptive variants include nonsense, frameshift, splice site, and missense mutations); UW-SNV: UW study participants with disruptive SNVs to DYRK1A

\section{Acknowledgements}

We would like to thank our funding sources and the families who took part in this study.

\section{Funding}

This research was supported by the National Institute for Mental Health, $\#$ R01MH100047 to R.A.B. and \#R01MH101221 to E.E.E. E.E.E. is an investigator of the Howard Hughes Medical Institute.

\section{Availability of data and materials}

The datasets used and analyzed during the current study are available from the corresponding author on reasonable request.

\section{Authors' contributions}

RAB and EEE developed the study concept. EEE and TT performed genetic sequencing, and $T T$ consulted on the presentation of gene variant data. Phenotypic data collection was carried out by RKE, HCM, and JG. RKE drafted the manuscript, and $R A B, T, J G, C M H$, and EEE provided critical revisions. All authors approved the final version of the manuscript for submission.

\section{Ethics approval and consent to participate}

Written consent was obtained from participants, and all procedures were approved by the University of Washington Institutional Review Board.

\section{Consent for publication}

Written informed consent was obtained from the participants for publication of their individual details and accompanying images in this manuscript. The consent form is held by the authors and is available for review by the Editor-in-Chief.

\section{Competing interests}

The authors declare that they have no competing interests.

\section{Publisher's Note}

Springer Nature remains neutral with regard to jurisdictional claims in published maps and institutional affiliations.

\section{Author details}

${ }^{1}$ Department of Psychiatry and Behavioral Sciences, University of Washington, CHDD Box 357920, Seattle, WA 98195, USA. ²Department of Genome Sciences, University of Washington, Seattle, WA, USA. ${ }^{3}$ School of Medicine, University of Washington, Seattle, WA, USA. ${ }^{4}$ Howard Hughes Medical Institute, Seattle, WA, USA. ${ }^{5}$ Center on Human Development and Disability, University of Washington, Seattle, WA, USA.

Received: 30 June 2017 Accepted: 27 September 2017 Published online: 05 October 2017

References

1. King BH, Navot N, Bernier R, Webb SJ. Update on diagnostic classification in autism. Current opinion in psychiatry. 2014;27(2):105-9. doi:10.1097/yco. 0000000000000040. Epub 2014/03/04. PubMed PMID: 24441420

2. Lord C, Petkova E, Hus V, Gan W, Lu F, Martin DM, et al. A multisite study of the clinical diagnosis of different autism spectrum disorders. Arch Gen Psychiatry. 2012;69(3):306-13. doi:10.1001/archgenpsychiatry.2011.148. Epub 2011/11/09. PubMed PMID: 22065253; PubMed Central PMCID: PMCPMC3626112

3. Higdon R, Earl RK, Stanberry L, Hudac CM, Montague E, Stewart E, et al. The promise of multi-omics and clinical data integration to identify and target personalized healthcare approaches in autism spectrum disorders. Omics : a journal of integrative biology. 2015;19(4):197-208. doi:10.1089/omi.2015. 0020. Epub 2015/04/02. PubMed PMID: 25831060; PubMed Central PMCID: PMCPMC4389910

4. Jeste SS, Geschwind DH. Disentangling the heterogeneity of autism spectrum disorder through genetic findings. Nat Rev Neurol. 2014;10(2):7481. doi:10.1038/nrneurol.2013.278. Epub 2014/01/29. PubMed PMID: 24468882; PubMed Central PMCID: PMCPMC4125617

5. O'Roak BJ, Deriziotis P, Lee C, Vives L, Schwartz JJ, Girirajan S, et al. Exome sequencing in sporadic autism spectrum disorders identifies severe de novo mutations. Nat Genet. 2011;43(6):585-9. doi:10.1038/ng.835. Epub 2011/05/ 17. PubMed PMID: 21572417; PubMed Central PMCID: PMCPMC3115696

6. Iossifov I, O'Roak BJ, Sanders SJ, Ronemus M, Krumm N, Levy D, et al. The contribution of de novo coding mutations to autism spectrum disorder. Nature. 2014;515(7526):216-21. doi:10.1038/nature13908. Epub 2014/11/05. PubMed PMID: 25363768; PubMed Central PMCID: PMCPmc4313871

7. Pinto D, Pagnamenta AT, Klei L, Anney R, Merico D, Regan R, et al. Functional impact of global rare copy number variation in autism spectrum disorders. Nature. 2010;466(7304):368-72. doi:10.1038/nature09146. Epub 2010/06/10. PubMed PMID: 20531469; PubMed Central PMCID: PMCPMC3021798

8. Sanders SJ, He X, Willsey AJ, Ercan-Sencicek AG, Samocha KE, Cicek AE, et al. Insights into autism spectrum disorder genomic architecture and biology from 71 risk loci. Neuron. 2015;87(6):1215-33. doi:10.1016/j.neuron.2015.09. 016. Epub 2015/09/25. PubMed PMID: 26402605; PubMed Central PMCID: PMCPMC4624267

9. Vorstman JA, Staal WG, van Daalen E, van Engeland H, Hochstenbach PF, Franke $\mathrm{L}$. Identification of novel autism candidate regions through analysis of reported cytogenetic abnormalities associated with autism. Mol Psychiatry. 2006;11(1):1, 18-28. Epub 2005/10/06. PubMed PMID: 16205736. doi:10.1038/sj.mp.4001757.

10. Stessman HA, Bernier R, Eichler EE. A genotype-first approach to defining the subtypes of a complex disease. Cell. 2014;156(5):872-7. https://doi.org/ 10.1016/j.cell.2014.02.002. Epub 2014/03/04. PubMed PMID: 24581488; PubMed Central PMCID: PMCPMC4076166

11. Bernier R, Golzio C, Xiong B, Stessman HA, Coe BP, Penn O, et al. Disruptive CHD8 mutations define a subtype of autism early in development. Cell. 2014;158(2):263-76. doi:10.1016/j.cell.2014.06.017. Epub 2014/07/08. PubMed PMID: 24998929; PubMed Central PMCID: PMCPmc4136921

12. Helsmoortel C, Vulto-van Silfhout AT, Coe BP, Vandeweyer G, Rooms L, van den Ende J, et al. A SWI/SNF-related autism syndrome caused by de novo mutations in ADNP. Nat Genet. 2014;46(4):380-4. doi:10.1038/ng.2899. Epub 2014/02/18. PubMed PMID: 24531329; PubMed Central PMCID: PMCPmc3990853

13. van Bon BW, Coe BP, Bernier R, Green C, Gerdts J, Witherspoon K, et al. Disruptive de novo mutations of DYRK1A lead to a syndromic form of 
autism and ID. Mol Psychiatry. 2016;21(1):126-32. doi:10.1038/mp.2015.5. Epub 2015/02/25. PubMed PMID: 25707398; PubMed Central PMCID: PMCPMC4547916

14. O'Roak BJ, Vives L, Fu W, Egertson JD, Stanaway IB, Phelps IG, et al. Multiplex targeted sequencing identifies recurrently mutated genes in autism spectrum disorders. Science (New York, NY). 2012;338(6114):1619-22. doi:10.1126/science.1227764. Epub 2012/11/20. PubMed PMID: 23160955; PubMed Central PMCID: PMCPmc3528801

15. Bronicki LM, Redin C, Drunat S, Piton A, Lyons M, Passemard S, et al. Ten new cases further delineate the syndromic intellectual disability phenotype caused by mutations in DYRK1A. European journal of human genetics: EJHG. 2015;23(11):1482-7. doi:10.1038/ejhg.2015.29. Epub 2015/04/30. PubMed PMID: 25920557; PubMed Central PMCID: PMCPMC4613470

16. APA. Diagnostic and statistical manual of mental disorders. 5th ed. Washington, D.C: Author; 2013.

17. van Bon BW, Coe BP, BBA dV. In: Pagon RA, Adam MP, Ardinger HH, editors. DYRK1A-related intellectual disability syndrome. Seattle: University of Washington; 2015.

18. Tejedor F, Zhu XR, Kaltenbach E, Ackermann A, Baumann A, Canal I, et al. minibrain: a new protein kinase family involved in postembryonic neurogenesis in Drosophila. Neuron. 1995;14(2):287-301. Epub 1995/02/01. PubMed PMID: 7857639

19. Fotaki V, Dierssen M, Alcantara S, Martinez S, Marti E, Casas C, et al. Dyrk1A haploinsufficiency affects viability and causes developmental delay and abnormal brain morphology in mice. Mol Cell Biol. 2002;22(18):6636-47. Epub 2002/08/23. PubMed PMID: 12192061; PubMed Central PMCID: PMCPMC135639

20. Fotaki V, Martinez De Lagran M, Estivill X, Arbones M, Dierssen M. Haploinsufficiency of Dyrk1A in mice leads to specific alterations in the development and regulation of motor activity. Behav Neurosci. 2004;118(4): 815-21. doi:10.1037/0735-7044.118.4.815. Epub 2004/08/11. PubMed PMID: 15301607

21. Ji J, Lee H, Argiropoulos B, Dorrani N, Mann J, Martinez-Agosto JA, et al. DYRK1A haploinsufficiency causes a new recognizable syndrome with microcephaly, intellectual disability, speech impairment, and distinct facies. European journal of human genetics : EJHG. 2015;23(11):1473-81. doi:10. 1038/ejhg.2015.71. Epub 2015/05/07. PubMed PMID: 25944381; PubMed Central PMCID: PMCPMC4613469

22. Luco SM, Pohl D, Sell E, Wagner JD, Dyment DA, Daoud H. Case report of novel DYRK1A mutations in 2 individuals with syndromic intellectual disability and a review of the literature. BMC medical genetics. 2016;17:15. doi:10.1186/s12881-016-0276-4. Epub 2016/02/29. PubMed PMID: 26922654; PubMed Central PMCID: PMCPMC4769499

23. Moller RS, Kubart S, Hoeltzenbein M, Heye B, Vogel I, Hansen CP, et al. Truncation of the Down syndrome candidate gene DYRK1A in two unrelated patients with microcephaly. Am J Hum Genet. 2008;82(5):1165-70. doi:10.1016/j.ajhg.2008.03.001. Epub 2008/04/15. PubMed PMID: 18405873; PubMed Central PMCID: PMCPMC2427221

24. van Bon BW, Hoischen A, Hehir-Kwa J, de Brouwer AP, Ruivenkamp C Gijsbers AC, et al. Intragenic deletion in DYRK1A leads to mental retardation and primary microcephaly. Clin Genet. 2011;79(3):296-9. doi:10.1111/j.13990004.2010.01544.x. Epub 2011/02/08. PubMed PMID: 21294719

25. Fraser FC, Sadovnick AD. Correlation of IQ in subjects with Down syndrome and their parents and sibs. J Ment Defic Res. 1976;20(3):179-82. Epub 1976/ 09/01. PubMed PMID: 135090

26. Malich S, Largo RH, Schinzel A, Molinari L, Eiholzer U. Phenotypic heterogeneity of growth and psychometric intelligence in Prader-Willi syndrome: variable expression of a contiguous gene syndrome or parentchild resemblance? Am J Med Genet. 2000;91 (4):298-304. Epub 2000/04/15. PubMed PMID: 10766987

27. Moreno-De-Luca A, Evans DW, Boomer KB, Hanson E, Bernier R, Goin-Kochel $\mathrm{RP}$, et al. The role of parental cognitive, behavioral, and motor profiles in clinical variability in individuals with chromosome 16p11.2 deletions. JAMA psychiatry. 2015;72(2):119-26. doi:10.1001/jamapsychiatry.2014.2147. Epub 2014/12/11. PubMed PMID: 25493922

28. Preece MA. The genetic contribution to stature. Horm Res. 1996;45(Suppl 2): 56-8. Epub 1996/01/01. PubMed PMID: 8805046

29. Lonsdale J, Thomas J, Salvatore M, Phillips R, Lo E, Shad S, Foster B. The genotype-tissue expression (GTEx) project. Nat Genet. 2013;45(6):580-85. doi:10.1038/ng.2653.
30. Ruaud L, Mignot C, Guet A, Ohl C, Nava C, Heron D, et al. DYRK1A mutations in two unrelated patients. European journal of medical genetics. 2015;58(3):168-74. doi:10.1016/j.ejmg.2014.12.014. Epub 2015/02/03. PubMed PMID: 25641759

31. Iglesias A, Anyane-Yeboa K, Wynn J, Wilson A, Truitt Cho M, Guzman E, et al. The usefulness of whole-exome sequencing in routine clinical practice. Genetics in medicine : official journal of the American College of Medical Genetics. 2014;16(12):922-31. doi:10.1038/gim.2014.58. Epub 2014/06/06. PubMed PMID: 24901346

32. Okamoto N, Miya F, Tsunoda T, Kato M, Saitoh S, Yamasaki M, et al. Targeted next-generation sequencing in the diagnosis of neurodevelopmental disorders. Clin Genet. 2015;88(3):288-92. doi:10.1111/cge.12492. Epub 2014/08/27. PubMed PMID: 25156961

33. Bartsch $\mathrm{O}$, Hinkel GK, Petersen MB, Konig U, Bugge M, Mikkelsen M, et al. A large family with subtelomeric translocation t (18;21)(q23;q22.1) and molecular breakpoint in the Down syndrome critical region. Hum Genet. 1997:100(5-6):669-75. Epub 1997/10/28. PubMed PMID: 9341890

34. Courcet JB, Faivre L, Malzac P, Masurel-Paulet A, Lopez E, Callier P, et al. The DYRK1A gene is a cause of syndromic intellectual disability with severe microcephaly and epilepsy. J Med Genet. 2012;49(12):731-6. doi:10.1136/ jmedgenet-2012-101251. Epub 2012/10/27. PubMed PMID: 23099646

35. Fujita H, Torii C, Kosaki R, Yamaguchi S, Kudoh J, Hayashi K, et al. Microdeletion of the Down syndrome critical region at 21q22. Am J Med Genet A. 2010;152a(4):950-3. doi:10.1002/ajmg.a.33228. Epub 2010/04/02. PubMed PMID: 20358607

36. Matsumoto N, Ohashi H, Tsukahara M, Kim KC, Soeda E, Niikawa N. Possible narrowed assignment of the loci of monosomy 21-associated microcephaly and intrauterine growth retardation to a 1.2-Mb segment at 21q22.2. Am J Hum Genet. 1997;60(4):997-9. Epub 1997/04/01. PubMed PMID: 9106547; PubMed Central PMCID: PMCPMC1712454

37. Oegema R, de Klein A, Verkerk AJ, Schot R, Dumee B, Douben $H$, et al. Distinctive phenotypic abnormalities associated with submicroscopic 21 q22 deletion including DYRK1A. Molecular syndromology. 2010;1(3):113-20. doi:10.1159/000320113. Epub 2010/10/30. PubMed PMID: 21031080 PubMed Central PMCID: PMCPMC2957846

38. Valetto A, Orsini A, Bertini V, Toschi B, Bonuccelli A, Simi F, et al. Molecular cytogenetic characterization of an interstitial deletion of chromosome 21 (21q22. 13q22.3) in a patient with dysmorphic features, intellectual disability and severe generalized epilepsy. European journal of medical genetics. 2012;55(5):362-6. doi:10.1016/.ejmg.2012.03.011. Epub 2012/05/03. PubMed PMID: 22548977

39. Yamamoto T, Shimojima K, Nishizawa T, Matsuo M, Ito M, Imai K. Clinical manifestations of the deletion of Down syndrome critical region including DYRK1A and KCNJ6. Am J Med Genet A. 2011;155a(1):113-9. doi:10.1002/ ajmg.a.33735. Epub 2011/01/05. PubMed PMID: 21204217

40. Fischbach GD, Lord C. The Simons Simplex Collection: a resource for identification of autism genetic risk factors. Neuron. 2010;68(2):192-5. doi:10. 1016/j.neuron.2010.10.006. Epub 2010/10/20. PubMed PMID: 20955926

41. Roche AF, Mukherjee D, Guo SM, Moore WM. Head circumference reference data: birth to 18 years. Pediatrics. 1987;79(5):706-12. Epub 1987/05/01. PubMed PMID: 3575026

42. Elliott CD. Differential Abilities Scales. 2nd ed. San Antonio: Harcourt Assessment; 2007

43. Wechsler D, Hsiao-pin C. Wechsler abbreviated scale of intelligence. 2nd ed. San Antonio: Pearson; 2011.

44. Sparrow SS, Balla DA, Ciccheti DV, Doll EA. Vineland-II: Vineland adaptive behavior scales: survey forms manual. Circle Pines: AGS Publishing; 2005.

45. Lord C, Rutter M, DiLavore PC, Risi S. Autism Diagnostic Observation Schedule-WPS (ADOS-WPS). Los Angeles: Western Psychological Services; 1999.

46. Lord C, Rutter M, DiLavore PC, Risi S, Gotham K, Bishop SL. Autism Diagnostic Observation Schedule--Second Edition. 2nd ed. Los Angeles: Western Psychological Services; 2013.

47. Lord C, Rutter M, Le Couteur A. Autism Diagnostic Interview--Revised: a revised version of a diagnostic interview for caregivers of individuals with possible pervasive developmental disorders. J Autism Dev Disord. 1994; 24(5):659-85. Epub 1994/10/01. PubMed PMID: 7814313

48. Constantino JN, Gruber CP. Social Responsiveness Scale. 2nd ed. Los Angeles: Western Psychological Services; 2012.

49. Christensen DL, Baio J, Van Naarden BK, Bilder D, Charles J, Constantino JN, et al. Prevalence and characteristics of autism spectrum disorder among children aged 8 years--Autism and Developmental Disabilities Monitoring Network, 11 Sites, United States, 2012. Morbidity and mortality weekly 
report Surveillance summaries (Washington, DC : 2002). 2016;65(3):1-23. 10. 15585/mmwr.ss6503a1. Epub 2016/04/01. PubMed PMID: 27031587

50. Hudac CM, Kresse A, Aaronson B, DesChamps TD, Webb SJ, Bernier RA, et al. Modulation of mu attenuation to social stimuli in children and adults with 16p11.2 deletions and duplications Exploring the heterogeneity of neural social indices for genetically distinct etiologies of autism. (1866-1947 (Print)). D - NLM: PMC4514956 OTO - NOTNLM.

51. Hudac CM, Stessman HAF, DesChamps TD, Kresse A, Faja S, Neuhaus E, et al. Exploring the heterogeneity of neural social indices for genetically distinct etiologies of autism. (1866-1947 (Print)).

52. Clark PJ. The heritability of certain anthropometric characters as ascertained from measurements of twins. 1955(0002-9297 (Print)). D - CLML: 5630:10388 D - NLM: PMC1716621 OTO - NLM.

53. Weaver DD, Christian JC. Familial variation of head size and adjustment for parental head circumference. J Pediatr. 1980;96(6):990-4. doi:10.1016/500223476(80)80623-8

54. Kent WJ, Sugnet CW, Furey TS, Roskin KM, Pringle TH, Zahler AM, et al. The human genome browser at UCSC. Genome Res. 2002;12(6):996-1006.

Submit your next manuscript to BioMed Central and we will help you at every step:

- We accept pre-submission inquiries

- Our selector tool helps you to find the most relevant journal

- We provide round the clock customer support

- Convenient online submission

- Thorough peer review

- Inclusion in PubMed and all major indexing services

- Maximum visibility for your research

Submit your manuscript at www.biomedcentral.com/submit
Biomed Central 